\title{
CFSE: A New Method for Identifying Human Limbal Stem Cells and Following Their Migration in Human Cornea
}

\author{
CHIARA BONZANO ${ }^{1}$, BARBARA CANCIANI ${ }^{2}$, SARA OLIVARI ${ }^{1}$, MARINA PAPADIA ${ }^{3}$, \\ ALESSANDRO BAGNIS ${ }^{1}$, CARLO ALBERTO CUTOLO ${ }^{1}$, ELISABETTA BONZANO ${ }^{4}$, \\ PAOLA PAGANI ${ }^{1}$, RANIERI CANCEDDA ${ }^{2}$ and CARLO ENRICO TRAVERSO ${ }^{1}$ \\ ${ }^{1}$ Eye Clinic, DiNOGMI, University of Genoa and IRCCS San Martino Polyclinic Hospital, Genoa, Italy; \\ ${ }^{2}$ Laboratory of Regenerative Medicine, Department of Oncology, \\ Biology and Genetics, IRCCS San Martino Polyclinic Hospital, Genoa, Italy; \\ ${ }^{3}$ Italian Auxologic Institute, Milan, Italy; \\ ${ }^{4}$ School of Experimental Medicine, University of Pavia and Department of Radiation Oncology, \\ IRCCS San Matteo Polyclinic Foundation, Pavia, Italy
}

\begin{abstract}
Aim: To develop a method capable of identifying human corneal limbal stem cells (LSCs) and follow their proliferation and migration in the epithelium. Materials and Methods: Ten fresh matched pairs of cadaveric normal human corneas were obtained from donors. Carboxyfluorescein diacetate succinimidyl ester (CFSE) was used to target LSCs. The distribution of CFSE-positive cell clusters was analyzed by fluorescence microscopy by counterstaining with 4',6diamidino-2-phenylindole (DAPI). Fluorescence was digitally recorded for seven days, and the rate of cell movement was determined. Results: CFSE-labeled cells were tracked in corneas. Analysis of time sequences revealed that they moved centripetally. Daily average CFSE-labeled LSC movement was $0.073 \pm 0.01 \mathrm{~cm}( \pm S D)$. Conclusion: CFSE allowed us to identify LSCs and to track their centripetal migration from the limbal basal layer to the anterior ocular surface. This experimental system appears to be a valuable tool for further studies on corneal epithelial cell migration and proliferation.
\end{abstract}

The corneal epithelium is a rapidly proliferating tissue in which cells are constantly renewed and lost. The natural turnover of human corneal epithelial cells takes place

This article is freely accessible online.

Correspondence to: Chiara Bonzano, MD, FEBO, Ophthalmology Clinic, DiNOGMI, University of Genoa and IRCCS San Martino Polyclinic Hospital, Viale Benedetto XV 5, 16132 Genoa, Italy. Tel.: +39 3381148751, e-mail: oculistabonzano@gmail.com

Key Words: Corneal stem cells, corneal epithelium, limbal stem cells, LSCs, carboxyfluorescein diacetate succinimidyl ester, CFSE, fluorescence microscopy. wherein superficial cells are evenly shed from the corneal surface by regular desquamation. These cells are constantly replaced by a population of stem cells, which reside in the basal limbal region and continue to cycle slowly during the entire lifetime (1). When these limbal stem cells (LSCs) are lost, the corneal epithelium is unable to repair and renew itself (2). Transient amplifying cells are LSC daughter cells and migrate centripetally into the basal layer of the corneal epithelium, where they differentiate toward the upper layers to become postmitotic cells (3). This mechanism of corneal maintenance, the X, Y, Z hypothesis (4), has been widely accepted and combined with the general assumption that corneal epithelial stem cells principally reside in the basal layer of the highly specialized and protected limbal niche (1, $5,6)$. The radial structure of the limbus is also known as the Palisades of Vogt (7). LSCs are a subpopulation of cells that are slow-cycling, poorly differentiated and highly proliferative $(8,9)$. It is broadly assumed that the central corneal epithelium has no stem cells or stem cell-like cells, instead, the central epithelium retains some level of regenerative ability $(3,10,11)$. Therefore the cells have little proliferative capacity compared to peripheral epithelial cells and LSCs (12). The continual renewal of the epithelium is fundamental to maintaining its normal functions, and a good understanding of cellular mechanisms is essential in comprehending pathological conditions such as persistent epithelium defect and wound healing.

Cell migration is one of the most important aspects of epithelial homeostasis, and despite a constant increase in knowledge in this area, there are not many investigations concerning the physiological migration of epithelial cells in the normal cornea, presumably because no appropriate methodology was available to detect cell movement. Although histological studies are not always suitable for the investigation 
of dynamic events, localization of typical cells during a time sequence can be seen after fluorescence labeling. Accordingly, we sought to establish a human model in which the migration of epithelial cells could be studied in healthy and uninjured cornea. To the best of our knowledge, although there are various fluorescein-based dyes used to label cells for in vivo migration studies (13-15), fluorescent labeling has never been used to track migration and proliferation of corneal epithelial cells before. Among others, a very useful intracellular fluorescent dye is carboxyfluorescein diacetate succinimidyl ester (CFSE). Its capacity to label cell populations with a high fluorescent intensity of exceptionally low variance in combination with its low cell toxicity, render it an ideal dye for measuring cell division. Several studies in literature showed that CFSE is an effective and popular means of monitoring cell division but to date, it has only been used to examine lymphocyte migration (16-18). In our study, we introduce a new method for identifying LSCs and tracking their centripetal migration from the limbal basal layer toward the ocular surface using CFSE.

\section{Materials and Methods}

Human corneal samples. Approval for all human tissue-based research was obtained from the local Ethics Committee (approval no.15490). Ten fresh matched pairs of cadaveric human corneas were collected from donors sourced through the Melvin Jones Eye Bank (Genoa, Italy). Corneas used in our study were unsuitable for transplantation since the cause of death was unknown, or there was an underlying systemic disease in the donor. Corneas were resected from the globe by cutting around the limbus to leave a 2- to 3-mm scleral margin and were then transported in Melvin Jones Eye Bank medium.

Explanted corneas were stored in complete minimum essential medium (MEM) (composed of minerals, amino acids, vitamins, and antibiotics) at $31^{\circ} \mathrm{C}$ to keep epithelial cells alive.

Histology. Corneas were fixed in $10 \%$ formalin buffered solution for 4 hours at room temperature. Following the fixation, the corneas were rinsed in water three times, $10 \mathrm{~min}$ each, dehydrated in a graded ethanolic series ( $70 \%$ to absolute), and embedded in paraffin. To determine the best plane of sectioning, one cornea was cut longitudinally, another one transversally. Sections were cut by a rotary microtome (Leica RM2165; Leica Microsystems, Cambridge, $\mathrm{UK})$ at $5 \mu \mathrm{m}$ thickness, and stained with hematoxylin/eosin stain. Briefly, the sections were deparaffinized in xylene, three times 10 min each, hydrated in a graded ethanolic series (from absolute to $70 \%$ ethanol) and then in bi-distilled water. Sections were stained first with hematoxylin solution (Bio-Optica, Milan, Italy) for $8 \mathrm{~min}$ and rinsed in running tap water for $10 \mathrm{~min}$, then with eosin $\mathrm{Y}$ solution (Carlo Erba, Milan, Italy). After that, the sections were differentiated and dehydrated by passage in $95 \%$ absolute ethanol quickly, then in xylene three times for 5 min each and sealed with a coverslip. An Axiovert $200 \mathrm{M}$ microscope was used to acquire images (Zeiss, Germany).

Epithelial stem cell labeling. On the first day, the corneas were incubated with CFSE [Vinci-Biochem, Vinci (Firenze) Italy] solution at $1 \mu \mathrm{l} / \mathrm{ml}$ in phosphate-buffered saline (PBS) diluted in

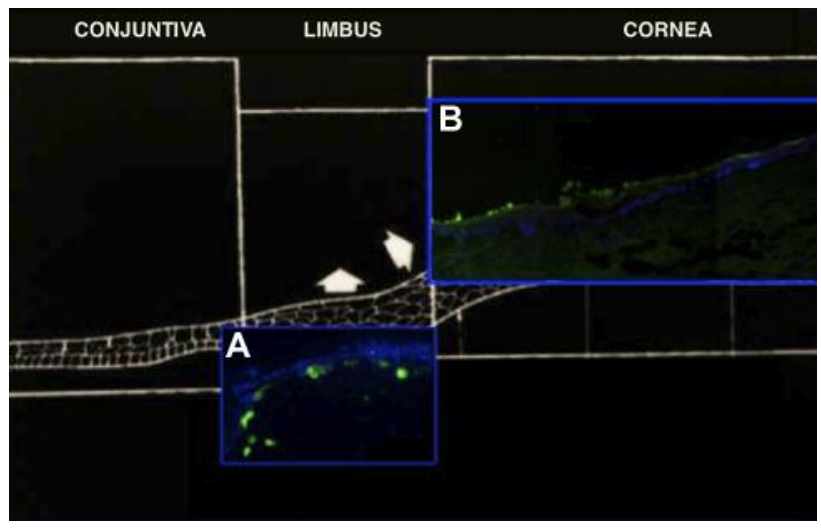

Figure 1. $X, Y, Z$ hypothesis demonstrated in a section of human cornea. Nuclei were counterstained with 4',6-diamidino-2-phenylindole (DAPI; blue). Carboxyfluorescein diacetate succinimidyl ester (CFSE)-labeled cells (green) showed centripetal migration of cells from the limbal basal layer toward the central cornea. Brighter CFSE labelling was present in the basal layer of the limbus (at T1) (A; magnification: 40X) where stem cells reside and CFSE was observed in the central epithelium (at T7) (B; magnification: 10x).

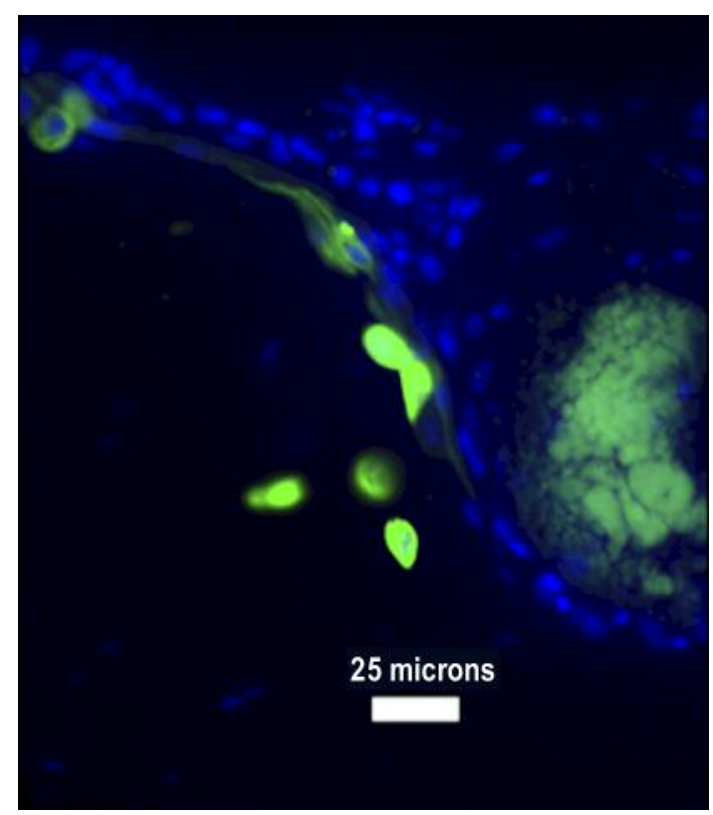

Figure 2. Stem cells in the basal limbal niche. Clusters of several highly fluorescent carboxyfluorescein diacetate succinimidyl ester-labeled cells in the basal limbal niche.

MEM for $30 \mathrm{~min}$ at $31^{\circ} \mathrm{C}$. After CFSE incorporation, the corneas were rinsed in a jar containing PBS for $5 \mathrm{~min}$ at $31^{\circ} \mathrm{C}$. Immediately after, the corneas were transferred to MEM at $31^{\circ} \mathrm{C}$. Each cornea was fixed at a defined time point starting from T0 (immediately after CFSE incorporation) through T1, T2, T3, T4, T5, T6, T7, where each time point was at $24-\mathrm{h}$ intervals. For the fixation, the 

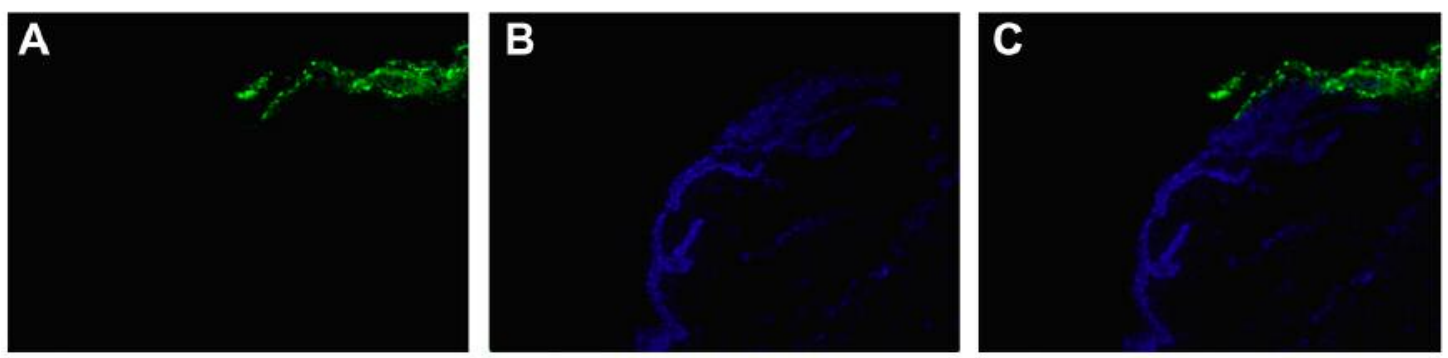

Figure 3. The distribution of the fluorescein-based dyes in corneal sections. 4',6-Diamidino-2-phenylindole stained all corneal cell nuclei (A), while carboxyfluorescein diacetate succinimidyl ester stained only the cytoplasm of corneal stem cells $(B)$. In $(C)$, both stains were used (magnification: 10x).

corneas were transferred in $10 \%$ formalin buffered solution for $4 \mathrm{~h}$ at room temperature and then they were washed in PBS three-time 5 min each. After three washes, each lasting $10 \mathrm{~min}$ in bi-distilled water, the corneas were stored in $20 \%$ sucrose solution in PBS at $4^{\circ} \mathrm{C}$ for a minimum of $24 \mathrm{~h}$.

Immunofluorescence. Corneas were cut with a scalpel into two halves, passing through the center of the cornea to prepare the samples for cutting with a cryostat. Few passages were required to embed the corneas for cryosectioning. Firstly, the samples were immersed in $20 \%$ sucrose solution plus cryostat embedding medium (Killik, Bio-Optica, Milan, Italy) $1: 1$ for $3 \mathrm{~h}$ on a shaker at $4^{\circ} \mathrm{C}$. Subsequently they were immersed in $20 \%$ sucrose solution plus cryostat embedding medium at $3: 1$ for $30 \mathrm{~min}$ on a shaker at $4{ }^{\circ} \mathrm{C}$ and finally embedded in $20 \%$ sucrose solution plus cryostat embedding medium $3: 1$, on dry ice. Corneas were kept at $-80^{\circ} \mathrm{C}$ before cutting. Samples included all layers of the cornea and were cut with a cryostat (Leica) at $8-\mu \mathrm{m}$ thickness, obtaining a cross section of the tissue. Slides were sealed with a coverslip, using Vectashield mounting media containing 4',6-diamidino-2phenylindole (DAPI) (Vinci-Biochem). The slides were analyzed, and images were acquired under an Axiovert $200 \mathrm{M}$ fluorescence microscope (Zeiss). Fluorescence was digitally recorded for 7 consecutive days (T1-T7), and the rate of cell movement was determined by tracing the different positions of CFSE-labeled LSCs using the manual tracking plugin in ImageJ.

\section{Results}

To investigate limbal epithelial cell movement, we developed a method which can identify the stem cells of human corneal epithelium and allows the proliferation and subsequent migration to be followed.

CFSE staining in the corneal epithelium resulted in a mosaic pattern of CFSE-positive cells, with the brightest cells present in the basal and suprabasal layer of the epithelium (highly fluorescent CFSE-labeled cells). At each subsequent division, the fluorescence level halved (low-level fluorescent CFSE-labeled cells), both highly and low-level fluorescent CFSE-labeled cells formed a fluorescent rim toward the central cornea. Clusters of several highly fluorescent CFSE-labeled cells were tracked in the cornea,

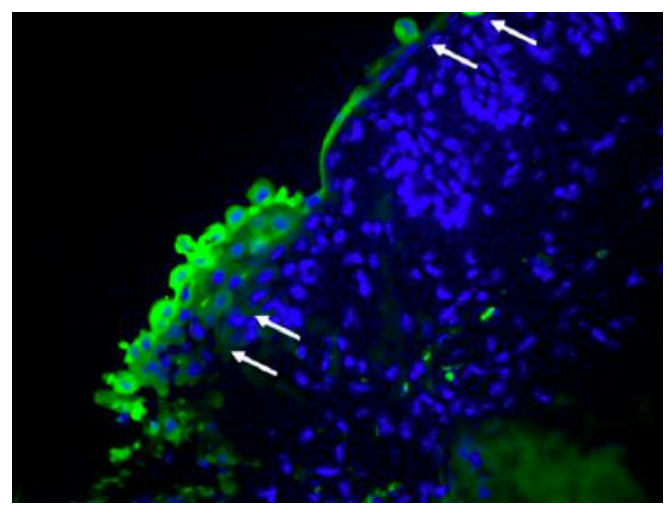

Figure 4. Carboxyfluorescein diacetate succinimidyl ester (CFSE)labeled cells. 4',6-Diamidino-2-phenylindole stained all corneal cell nuclei, while CFSE stained only the cytoplasm of corneal stem cells (arrows) (magnification: 40x).

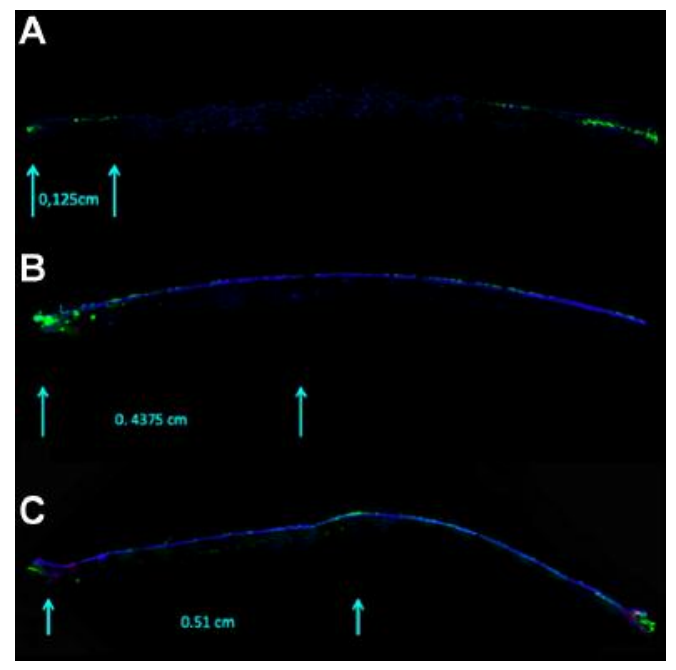

Figure 5. A representative time-lapse sequence of carboxyfluorescein diacetate succinimidyl ester-labeled cells tracked in corneas. Cell proliferation across the human cornea at T1 (A), T6 (B), and T7 (C) (magnification: 10x). 
and an analysis of time-lapse sequences revealed that they moved centripetally. CFSE and DAPI differential staining allowed highlighting of stem cells alone. Green fluorescence marked these cells (Figures 1-4).

A time-lapse sequence was digitally recorded. The mean \pm SD daily movement of CFSE-labeled cells was $0.073 \pm 0.01 \mathrm{~cm}$. The mean movement of CFSE-labeled LSCs on each day was: T1: $0.125 \mathrm{~cm}$; T2: $0.188 \mathrm{~cm}$; T3: $0.250 \mathrm{~cm}$; T4: $0.312 \mathrm{~cm}$; T5: $0.375 \mathrm{~cm}$; T6: $0.438 \mathrm{~cm}$; and T7: $0.510 \mathrm{~cm}$ (Figure 5).

CFSE staining allowed us to track corneal epithelial cells from the limbal basal layer centripetally to the superficial epithelium and was shown to be a reliable method allowing observation and quantification of the migration of epithelial cells on a daily basis. CFSE-labeled cells moved from the limbus towards the corneal surface in 1 week on average.

The use of CFSE staining followed by fluorescence microscopic observation allowed us to analyze the movement of epithelial cells in a normal human cornea.

\section{Discussion}

In the present study, we demonstrated the use of fluorescent microscopy to study migration of LSCs in the human cornea. No direct methods have been well established yet to identify corneal stem cells due to the lack of specific molecular markers, even if different putative stem cell markers have been proposed (19-21). In the late 1980s, Parish and Weston made a concerted effort to identify new fluorescent dyes that showed more persistent staining properties and different spectral characteristics to $\mathrm{H} 33342$ (18), the most valuable dye for studying cell migration as yet. A wide range of membrane-permeant, fluorescein-based dyes, to measure intracellular $\mathrm{pH}$, intracellular ion concentrations, intercellular adhesion and general cell viability, have become commercially available from Molecular Probes (Eugene, OR, USA). Today these seem ideal for use in labeling cells for in vivo migration studies. A key feature of these dyes is that they are coupled with acetate or acetoxymethyl side chains, which make them much more membrane-permeant. The side chains are esterase-sensitive and therefore when the dyes enter the cell, endogenous intracellular esterases remove the acetate/acetoxymethyl groups, and the dyes are trapped inside cells. The stable incorporation of intracellular fluorescent dye into cells provides an innovative tool for monitoring cell migration. Since the fluorescent labeling remains in daughter cells, it allows subsequent cell cycles to monitored (16).

On the basis of these observations, we chose CFSE both for these properties and for its lack of toxicity (22). To the best of our knowledge CFSE fluorescent labeling has never been used before to track migration and proliferation corneal epithelial cells. In our study, CFSE was used to target corneal epithelial cells. The shape and distribution of CFSE-positive cell clusters and cell motion were analyzed in frozen cross-sections via fluorescence microscopy by counterstaining with DAPI. DAPI is a blue-fluorescent DNA stain that exhibits 20 -fold enhancement of fluorescence upon binding to AT regions of double-stranded DNA $(23,24)$. It is excited by the violet (405 $\mathrm{nm}$ ) laser line (25) and is used as a nuclear counterstain in fluorescence microscopy. It is usually used to stain fixed cells since the dye is cell impermeant, but it can also enter live cells if used at higher concentrations (26). Fluorescence was digitally recorded for 7 consecutive days, and the rate of cell movement was determined following the different position of the CFSE-labeled LSCs.

The use of CFSE in human corneas allowed us to identify LSCs and track their centripetal migration from the limbal basal layer to the epithelial surface using fluorescence microscopy. It provided us with a reliable method with the ability to check epithelial proliferation on a daily basis. We found that the best plane of sectioning was longitudinal because only such sections allowed observation of all the corneal layers. This experimental system paves the way for further research on epithelial cell migration in the normal cornea and in helping to better understand corneal epithelium dynamics. The protocol presented above is well established for the healthy human cornea, but could equally be applied to cornea affected by various conditions, such as corneas that had been exposed to chronic topical medications, in order to show possible changes in cell behavior.

It is likely that additional fluorescent dyes with different spectral properties from those of CFSE will become available for proliferation and tracking studies of limbal cells in the future. We trust that our experiment will trigger further developments in this area.

\section{Conflicts of Interest}

There is no conflict of interest to disclose in regard to this study.

\section{Authors' Contributions}

C.B. and E.B. conceived the original idea. C.B., B.C., M.P. conceived and planned the experiments. C.B., S.O. and B.C. carried out the experiments. P.P. contributed to sample preparation. C.B., B.C., M.P., A.B. contributed to the interpretation of the results. C.B. and E.B. took the lead in writing the article. C.B., C.C. and E.B. processed the experimental data, performed the analysis, drafted the article and designed the figures. R.C. and C.T. supervised the project. All authors provided critical feedback and helped shape the research, analysis and article.

\section{Acknowledgements}

The Authors are grateful to the Lions Melvin Jones Eye Bank (Genoa, Italy), and especially to Dr. Nadia Randazzo, for supplying donor corneas. 


\section{References}

1 Chang CY, Green CR, McGhee CN and Sherwin T: Acute wound healing in the human central corneal epithelium appears to be independent of limbal stem cell influence. Invest Ophthalmol Vis Sci 49: 5279-5286, 2008. PMID: 18515566. DOI: $10.1167 /$ iovs. $07-1260$

2 Kenyon KR and Tseng SC: Limbal autograft transplantation for ocular surface disorders. Ophthalmology 96: 709-722; discussion 722-723, 1989. DOI: 10.1016/s0161-6420(89)32833-8

3 Lehrer MS, Sun TT and Lavker RM: Strategies of epithelial repair: Modulation of stem cell and transit amplifying cell proliferation. J Cell Sci 111(Pt 19): 2867-2875, 1998. PMID: 9730979.

4 Schermer A, Galvin S and Sun TT: Differentiation-related expression of a major $64 \mathrm{~K}$ corneal keratin in vivo and in culture suggests limbal location of corneal epithelial stem cells. J Cell Biol 103: 49-62, 1986. PMID: 2424919. DOI: 10.1083/ jcb.103.1.49

5 Dua HS, Shanmuganathan VA, Powell-Richards AO, Tighe PJ and Joseph A: Limbal epithelial crypts: A novel anatomical structure and a putative limbal stem cell niche. Br J Ophthalmol 89: 529-532, 2005. PMID: 15834076. DOI:10.1136/ bjo. 2004.049742

6 Boulton M and Albon J: Stem cells in the eye. Int J Biochem Cell Biol 36: 643-657, 2004. PMID: 15010329. DOI:10.1016/ j.biocel.2003.10.013

7 Goldberg MF and Bron AJ: Limbal palisades of Vogt. Trans Am Ophthalmol Soc 80: 155-171, 1982. PMID: 7182957.

8 Daniels JT, Dart JK, Tuft SJ and Khaw PT: Corneal stem cells in review. Wound Repair Regen Off Publ Wound Heal Soc Eur Tissue Repair Soc 9: 483-494, 2001. PMID: 11896990.

9 Wolosin JM, Xiong X, Schütte M, Stegman Z and Tieng A: Stem cells and differentiation stages in the limbo-corneal epithelium. Prog Retin Eye Res 19: 223-255, 2000. PMID: 10674709.

10 Dua HS and Azuara-Blanco A: Limbal stem cells of the corneal epithelium. Surv Ophthalmol 44: 415-425, 2000. PMID: 10734241.

11 Majo F, Rochat A, Nicolas M, Jaoude GA and Barrandon Y: Oligopotent stem cells are distributed throughout the mammalian ocular surface. Nature 456: 250-4, 2008. PMID: 18830243. DOI: 10.1038/nature07406.

12 Daniels JT, Harris AR and Mason C: Corneal epithelial stem cells in health and disease. Stem Cell Rev 2: 247-254, 2006. PMID: 17625261. DOI: 10.1007/s12015-006-0053-4

13 Day RN and Schaufele F: Fluorescent protein tools for studying protein dynamics in living cells: A review. J Biomed Opt 13: 031202, 2008. PMID: 18601526. DOI: 10.1117/1.2939093

14 Nolte MA, Kraal G and Mebius RE: Effects of fluorescent and nonfluorescent tracing methods on lymphocyte migration in vivo. Cytom Part J Int Soc Anal Cytol 61: 35-44, 2004. PMID: 15351987. DOI: 10.1002/cyto.a.20074
15 Weston SA and Parish CR: New fluorescent dyes for lymphocyte migration studies. Analysis by flow cytometry and fluorescence microscopy. J Immunol Methods 133: 87-97, 1990. PMID: 2212694. DOI: $10.1016 / 0022-1759(90) 90322-\mathrm{m}$

16 Parish CR, Glidden MH, Quah BJC and Warren HS: Use of the intracellular fluorescent dye CFSE to monitor lymphocyte migration and proliferation. Curr Protoc Immunol Chapter 4: Unit4.9, 2009. PMID: 19235770. DOI: 10.1002/047114 2735.im0409s84

17 Parish CR and Warren HS: Use of the intracellular fluorescent dye CFSE to monitor lymphocyte migration and proliferation. Curr Protoc Immunol Chapter 4: Unit 4.9, 2002. PMID: 18432883. DOI: $10.1002 / 0471142735$.im0409s49

18 Parish CR: Fluorescent dyes for lymphocyte migration and proliferation studies. Immunol Cell Biol 77: 499-508, 1999. PMID: 10571670. DOI:10.1046/j.1440-1711.1999.00877.x

19 Ahmad S, Figueiredo F and Lako M: Corneal epithelial stem cells: characterization, culture and transplantation. Regen Med 1: 29-44, 2006. PMID: 17465818. DOI: 10.2217/17460751.1.1.29

20 Schlötzer-Schrehardt $U$ and Kruse FE: Identification and characterization of limbal stem cells. Exp Eye Res 81: 247-264, 2005. PMID: 16051216. DOI: 10.1016/j.exer.2005.02.016

21 Chee KYH, Kicic A and Wiffen SJ: Limbal stem cells: The search for a marker. Clin Experiment Ophthalmol 34: 64-73, 2006. PMID: 16451261. DOI: 10.1111/j.1442-9071.2006. 01147.x

22 Quah BJC and Parish CR: The use of carboxyfluorescein diacetate succinimidyl ester (CFSE) to monitor lymphocyte proliferation. J Vis Exp: e2259, 2010. DOI: 10.3791/2259

23 Eriksson S, Kim SK, Kubista M and Nordén B: Binding of 4',6diamidino-2-phenylindole (DAPI) to AT regions of DNA: Evidence for an allosteric conformational change. Biochemistry 32: 29872998, 1993. PMID: 8457562. DOI: 10.1021/bi00063a009

24 Larsen TA, Goodsell DS, Cascio D, Grzeskowiak K and Dickerson RE: The structure of DAPI bound to DNA. J Biomol Struct Dyn 7: 477-491, 1989. PMID: 2627296. DOI: 10.1080/ 07391102.1989.10508505

25 Shaw AK and Pal SK: Fluorescence relaxation dynamics of acridine orange in nanosized micellar systems and DNA. J Phys Chem B 111: 4189-4199, 2007. PMID: 17394304. DOI: 10.1021/jp067156r

26 Biancardi A, Biver T, Secco F and Mennucci B: An investigation of the photophysical properties of minor groove bound and intercalated DAPI through quantum-mechanical and spectroscopic tools. Phys Chem Chem Phys 15: 4596-4603, 2013. PMID: 23423468. DOI: $10.1039 / \mathrm{c} 3 \mathrm{cp} 44058 \mathrm{c}$

Received August 3, 2019

Revised August 31, 2019

Accepted September 3, 2019 